\title{
Tapering body stiffness shortens upper gastrointestinal examination via transoral insertion with ultrathin endoscope
}

\section{(ㄷ)(우우}

Authors

Satoshi Ono, Shun Ito, Kyohei Maejima, Shosuke Hosaka, Kiyotaka Umeki, Shin-ichiro Sato

Institution

Department of Gastroenterology, Chiba-nishi General Hospital, 1-107, Kanegasaku, Matsudo, Chiba

submitted 8.4.2020

accepted after revision 18.5 .2020

\section{Bibliography}

Endoscopy International Open 2020; 08: E1748-E1753

DOI 10.1055/a-1266-3203

ISSN 2364-3722

(c) 2020. The Author(s).

This is an open access article published by Thieme under the terms of the Creative Commons Attribution-NonDerivative-NonCommercial License, permitting copying and reproduction so long as the original work is given appropriate credit. Contents may not be used for commecial purposes, or adapted, remixed, transformed or built upon. (https://creativecommons.org/licenses/by-nc-nd/4.0/)

\section{Corresponding author}

Satoshi Ono, MD, PhD, Department of Gastroenterology, Chiba-Nishi General Hospital, 1-107, Kanegasaku, Matsudo, Chiba 270-2251, Japan

Fax: +81-47-389-5162

satoshi-tky@umin.ac.jp

\section{ABSTRACT}

Background and study aims Ultrathin endoscopes are commonly used for surveillance esophagogastroduodenoscopy (EGD) to reduce discomfort associated with scope insertion. However, the flexibility of an ultrathin endoscope is a trade-off between reducing discomfort and lengthening examination time.

Patients and methods The EG17-J10 (EG17) is a novel ultrathin endoscope characterized by its tapering body stiffness; however, the flexibility of its tip is comparable to that of the traditional ultrathin endoscope EG16-K10 (EG16). We compared EGD examination time between EG17 and EG16. A total of 319 examinees who underwent EGD from November 2019 to January 2020 at the Chiba-Nishi General Hospital were enrolled. Six examinees were excluded due to past history of surgical resection of the upper gastrointestinal tract or too much food residues; 313 examinees (EG17, 209; EG16,104) were retrospectively analyzed. The examination time was divided into three periods: esophageal insertion time (ET), gastroduodenal insertion time (GDT), and surveillance time of the stomach (ST). The total amount of ET, GDT, and ST was defined as total examination time (TT).

Results TT of EGD using EG17 was significantly shorter compared to EGD using EG16 (222.7 \pm 68.9 vs. $245.7 \pm 78.5$ seconds) $(P=0.004)$. Among the three periods of examination time, ET $(66.7 \pm 24.1$ vs. $76.0 \pm 24.1$ seconds $)(P=$ $0.001)$ and GDT (47.9 \pm 17.4 vs. $55.2 \pm 35.2$ seconds $)(P=$ $0.007)$ of EGD using EG17 were significantly shorter compared to EGD using EG16, except for ST (108.1 \pm 51.5 .1 vs. $114.5 \pm 50.1$ seconds) $(P=0.148)$.

Conclusion An ultrathin endoscope with tapering body stiffness can shorten EGD examination time, mainly due to the shortening of insertion time.

\section{Introduction}

Endoscopy plays an important role in daily clinical practice of gastrointestinal diseases. High-resolution endoscopy with image-enhancement function has enabled the early detection of gastrointestinal neoplasms [1-4]. The magnifying function made it possible to accurately evaluate superficial neoplasms before resection [5,6]. In addition, endoscopic treatment has been adopted as a reliable, minimally invasive option for such superficial neoplasms [7-9]. As a result, application of endos- copy in this field is expanding. Concurrently, the importance of surveillance esophagogastroduodenoscopy (EGD) is increasing, despite the feeling of hesitation among examinees. Undoubtedly, one of the most important factors associated with this hesitation is the discomfort caused by the procedure itself.

An ultrathin endoscope, which can be inserted transorally or transnasally, was mainly developed with the aim of reducing patient discomfort $[10,11]$. EGD via transnasal insertion is associated with reduced gag reflex. However, due to its structural limitations, reduction of body stiffness is necessary. As a 


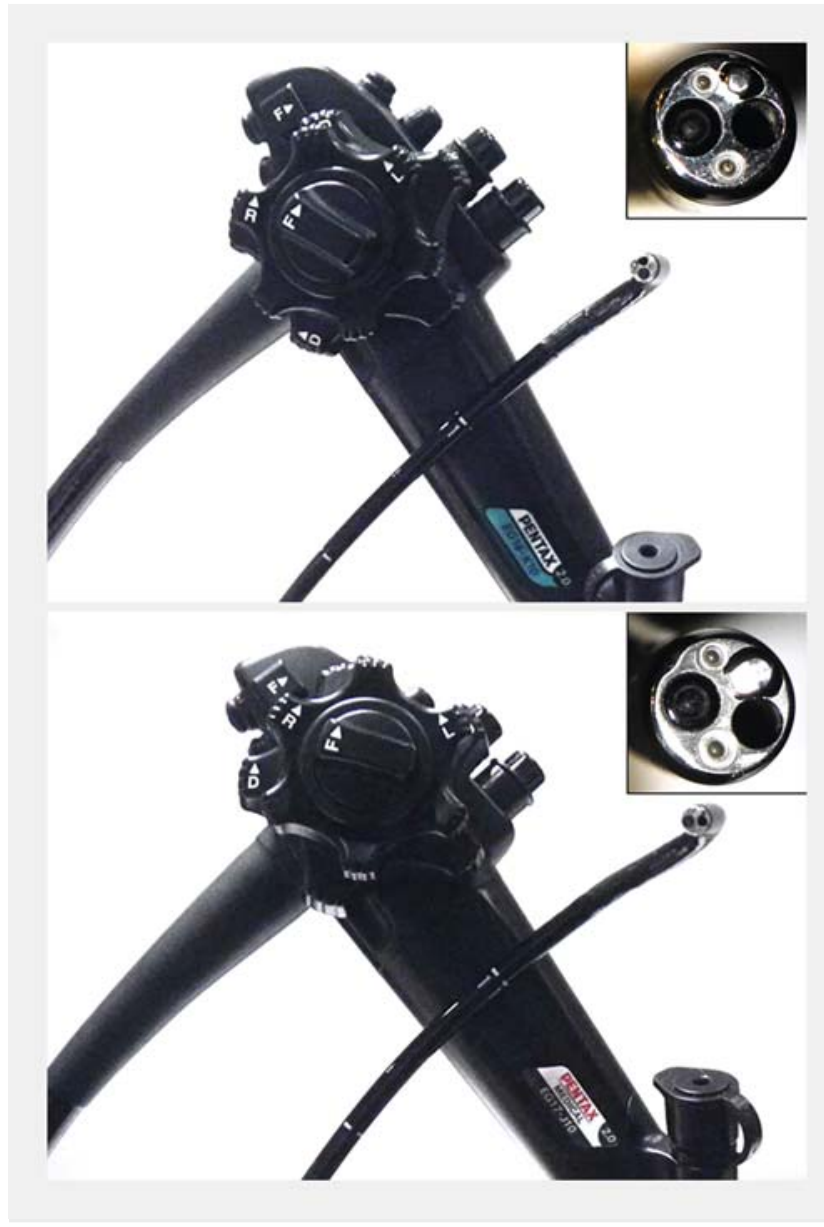

- Fig. 1 Image of the EG16-K10 and EG17-J10.

result, examination time using ultrathin scopes is longer than with conventional scopes. In a previous study, a flexible ultrathin scope was found to reduce patient discomfort during the examination but significantly lengthened the examination time [12]. From the standpoint of time efficiency, a longer ex- amination time is not preferable. Therefore, appropriate stiffness of the ultrathin endoscopes is mandatory.

The EG17-J10 (EG17) (PENTAX Medical, Hoya Corp., Tokyo, Japan) is a novel ultrathin scope characterized by tapering body stiffness compared with the previous generation model EG16-K10 (EG16) (PENTAX Medical, Hoya Corp., Tokyo, Japan). Its body, with the exception of the comparatively flexible tip, is designed to maintain strong stiffness ( $\mathbf{F i g . 1}$ ). In theory, it is expected to reduce examination time as mentioned above, but the real effect of the tapering body stiffness has not been evaluated in clinical daily practice. Therefore, this study aimed to evaluate the actual impact of the tapering body stiffness on EGD examination time using two types of ultrathin scopes.

\section{Patients and methods}

This was a retrospective, single-institution study conducted in Chiba-Nishi General Hospital, with approval from the ethics committee. Data were collected from digital records of 319 consecutive examinees who underwent surveillance EGD using the EG16 and EG17 from November 2019 to January 2020. During that time, the examinees were consecutively assigned to the two types of ultrathin scopes in a ratio of 1 to 2 , based on the number of scopes available in our institute.

The specifications of the EG16 and EG17 are summarized in - Table 1. Ex vivo values for insufflation-suction power were evaluated 10 times and are shown as mean \pm standard deviation. An endoscopic flexibility index (EFI) was evaluated ( Fig.2) [12] as follows. We fixed the middle portion of the endoscope to a flat surface and allowed the tip of the endoscope to bend freely under the influence of gravity. After adjusting the length of the endoscope from 150 to $400 \mathrm{~mm}$ to enable free movement under the influence of gravity, we mapped the position of the endoscope tip on a two-dimensional grid. The mean horizontal distances at fixed points of 200, 250, 300,350 , and $400 \mathrm{~mm}$ were used as an EFI.

In this study, we used an EPK-i7010 OPTIVISTA PLUS (PENTAX Medical, Hoya Corp., Tokyo, Japan) as a video processor

\begin{tabular}{|c|c|c|}
\hline & EG16-K10 & EG17-J10 \\
\hline Field of view $\left({ }^{\circ}\right)$ & 140 & 140 \\
\hline Tip deflection up/down $\left({ }^{\circ}\right)$ & $210 / 120$ & $210 / 120$ \\
\hline Tip deflection right/left $\left(^{\circ}\right)$ & $120 / 120$ & $120 / 120$ \\
\hline Diameter of distal end (mm) & 5.2 & 5.4 \\
\hline Diameter of insertion tube $(\mathrm{mm})$ & 5.4 & 5.7 \\
\hline Diameter of instrumental channel (mm) & 2.0 & 2.0 \\
\hline Suction speed (mL/sec) & $5.13 \pm 0.14$ & $5.41 \pm 0.23$ \\
\hline Insufflation speed (mL/sec) & $13.26 \pm 0.16$ & $21.60 \pm 0.28$ \\
\hline $\mathrm{EFI}(\mathrm{mm})$ & 148.7 & 177.5 \\
\hline
\end{tabular}




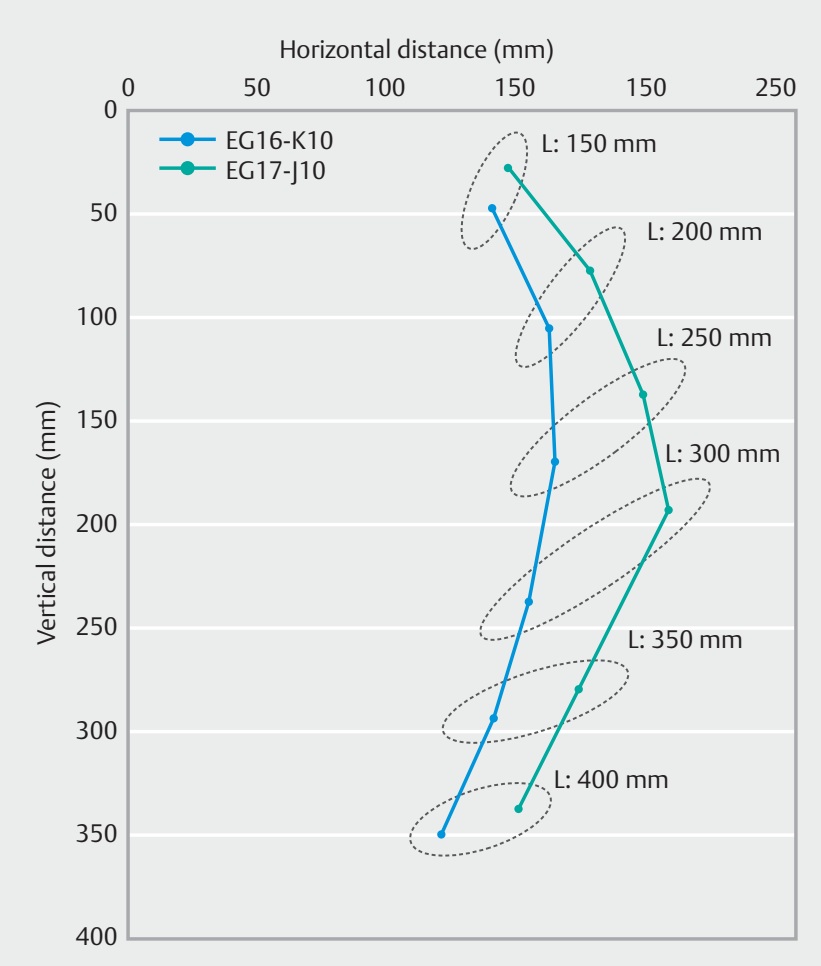

- Fig. 2 Two-dimensional plot of the transition from the tip of the two endoscopes. The length of the endoscope allowed free movement under the influence of gravity.

for the two scopes. The insufflation strength of the processor was configured to the strongest level. The suction tube was connected to the embedded suction pipe with stable suction power. These settings are similar with the ones we usually use for ultrathin scopes in our clinical daily practice.

All EGDs were performed via transoral insertion because of the clinical style of our institution. For preparation, we used pronase (PronaseMS; Kaken Pharmaceutical CO., Ltd. Tokyo, Japan) and 5-mL dimethicone 2\% (Gascon; Kissei Pharmaceutical Co., Ltd. Matsumoto, Japan), followed by five pushes of $8 \%$ lidocaine hydrochloride (Aspen Japan K.K., Tokyo, Japan) into the oral cavity. For examinees who underwent EGD under conscious sedation, we used 0.5 to $2.0 \mathrm{mg} / \mathrm{kg}$ propofol bolus infusion to keep the anesthesia level at -2 or -3 point of RASS during the examination [13]. Examinees with a past history of surgical resection of the upper gastrointestinal tract and those with too much food residue were excluded from the study.

All EGDs were performed by five well-trained endoscopists who experience performing more than 1,000 EGDs. Images were made at the following points during every EGD:

1. After insertion into the esophagogastric junction (EGJ), at the point of arrival at the EGJ.

2. After suctioning of fluid from the stomach and insertion of the scope through the stomach to the descending portion of duodenum, at the point of arrival at the duodenal papilla.

3. After the scope was pulled into the stomach (approximately 30 images).

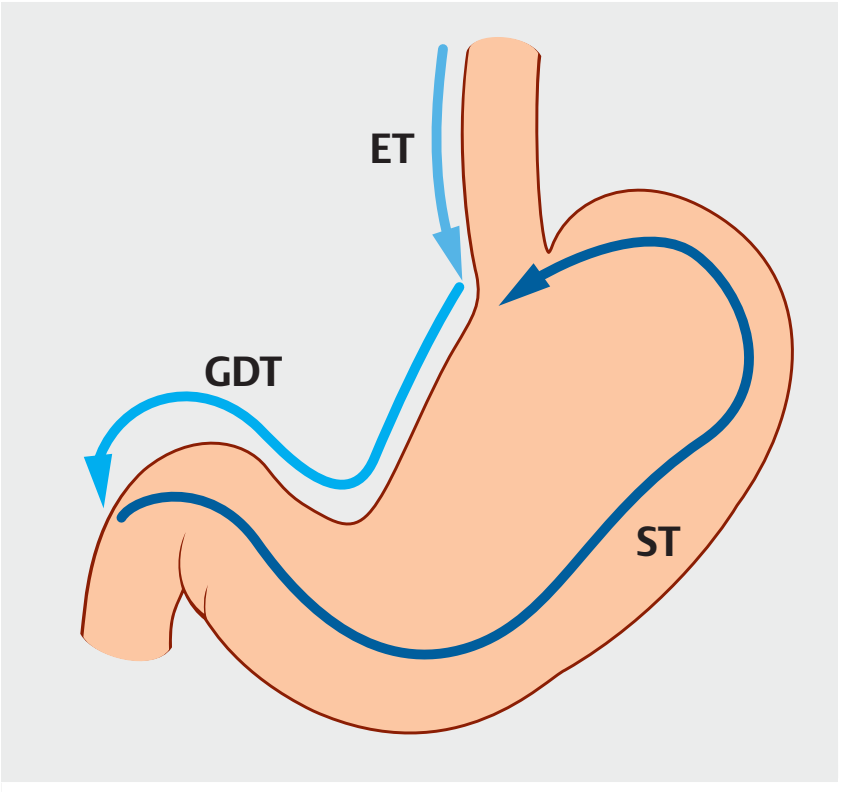

Fig. 3 The three examination periods.

4. After the scope was pulled back, to images were captured of the esophagus.

In cases where lesions were present, additional images were captured or biopsies taken.

Using the digital records of the images, we calculated the examination time and divided it into three periods: esophageal insertion time (ET), gastroduodenal insertion time (GDT), and surveillance time in the stomach (ST) ( ST combined was defined as the total examination time (TT). All biopsies were performed only for suspicious lesions. Random biopsies were not performed in this study.

Statistical analyses were performed using the student's $t$ test, $x 2$ test, and Fisher's exact test. All analyses were performed using R software (R Foundation for Statistical Computing, Vienna, Austria).

\section{Results}

Of 319 examinees, four were excluded due to a past history of surgical resection of the upper gastrointestinal tract and two due to too much food residue. A total of 313 examinees were assessed. The characteristics of the examinees are summarized in $>$ Table 2. No significant differences were found in age, sex, or use of sedation between the EG16 and EG17 groups.

Examination time also is summarized in $>$ Table 2. TT was approximately $10 \%$ shorter in the EG17 than in the EG16 group, although the total number of images was not significantly different between the groups. ET and GDT were significantly shorter in the EG17 than in the EG16 group. There was no significant difference in ST between the two groups.

We analyzed the learning effect by comparing the examinees in the first and second halves of the study. The effect of use of sedation was also analyzed, although no significant dif- 
- Table 2 Characteristics of examinees and summary of the results.

\begin{tabular}{|l|l|l|l|l|}
\hline & Total & EG16-K10 & EG17-J10 \\
\hline Number of examinees & 313 & 104 & 209 \\
\hline Age (years) & $63.1 \pm 13.4$ & $63.3 \pm 12.7$ & $63.0 \pm 13.8$ \\
\hline Sex (M/F) & $181 / 132$ & $64 / 40$ & $117 / 92$ \\
\hline Sedation (Y/N) & $208 / 105(66.6 \%)$ & $65 / 39(62.5 \%)$ & $143 / 66(68.4 \%)$ \\
\hline TT (sec) & $230.3 \pm 73.0$ & $245.7 \pm 78.5$ & $222.7 \pm 68.9$ \\
\hline ET (sec) & $69.8 \pm 24.5$ & $76.0 \pm 24.1$ & $66.7 \pm 24.1$ \\
\hline GDT (sec) & $50.3 \pm 25.0$ & $55.2 \pm 35.2$ & $47.9 \pm 17.4$ \\
\hline ST (sec) & $110.2 \pm 51.1$ & $114.5 \pm 50.1$ & $108.1 \pm 51.5$ \\
\hline Number of pictures & $36.2 \pm 6.5$ & $36.0 \pm 6.3$ & $36.4 \pm 6.6$ \\
\hline Biopsy (Y/N) & $36 / 277(11.5 \%)$ & $11 / 93(10.6 \%)$ & 0.458 \\
\hline TT, total examination time; ET, esophageal insertion time; GDT, gastroduodenal insertion time; ST, surveillance time in the stomach. \\
\hline
\end{tabular}

- Table 3 Comparison of examinees in the first and second halves of the study.

\begin{tabular}{|l|l|l|l|}
\hline & Early period & Late period & P value \\
\hline Number of examinees & 150 & 163 & 0.4877 \\
\hline Age (years) & $63.1 \pm 13.7$ & $63.1 \pm 13.2$ & 0.6076 \\
\hline Sex (M/F) & $84 / 66$ & $97 / 66$ & 0.3586 \\
\hline Sedation (Y/N) & $101 / 49(67.3 \%)$ & $107 / 56(65.6 \%)$ & 0.0451 \\
\hline Scope (EG16-K10/EG17-J10) & $41 / 109$ & $63 / 100$ & 0.4763 \\
\hline TT (sec) & $230.5 \pm 79.6$ & $230.1 \pm 66.3$ & 0.4285 \\
\hline ET (sec) & $70.0 \pm 25.9$ & $69.5 \pm 23.0$ & 0.1993 \\
\hline GDT (sec) & $51.6 \pm 31.3$ & $49.2 \pm 17.4$ & $111.4 \pm 49.0$ \\
\hline ST (sec) & $109.0 \pm 53.3$ & $37.2 \pm 6.5$ & 0.3392 \\
\hline Number of pictures & $35.2 \pm 6.3$ & $12 / 151$ & 0.0035 \\
\hline Biopsy (Y/N) & $24 / 126$ & 0.02672 \\
\hline TT, total examination time; ET, esophageal insertion time; GDT, gastroduodenal insertion time; ST, surveillance time in the stomach.
\end{tabular}

ferences were found ( $\triangleright$ Table 3 and $\triangleright$ Table 4 ). The effect of age was also analyzed, and revealed no significant difference in examination time for examinees over 60 years and under 60 years of age ( $\downarrow$ Table 5 ). However, analysis of age and scope type combined showed no significant differences between the two scopes only in STs of examinees over 60 years old ( $>$ Table 6 ).

\section{Discussion}

In the field of gastroenterology, ultrathin scopes are preferable due to reduced patient discomfort associated with surveillance EGD [14]. Regardless of the route of insertion route or patient age, ultrathin scopes facilitate low-discomfort EGD, although they are not preferred by operators because of the longer examination time associated with low maneuverability and insufflation-suction power $[11,12]$. The thinner body of these devi- ces results in a trade-off between reducing discomfort and their disadvantages. From this standpoint, the development of the ultrathin scopes is a dilemma.

In this study, we evaluated examination time to gauge the efficacy of a newly developed ultrathin scope. Insertion time into the EG] and duodenal papilla is shorter with the EG17. We speculate that the tapering body stiffness improves maneuverability, especially when pushing the scope. Moreover, the EG17 reduced ST for examinees under 60 years old, whereas no significant reduction in ST was seen in examinees over 60 years old. ST could be affected by various factors other than body stiffness. Among the examinees over 60 years old, cases of combined atrophic gastritis and hiatus hernia require longer examination time to survey the whole mucosa of the stomach while keeping the lumen expanded enough. In addition, the mucosa is often covered with mucus in cases of atrophic gastri- 
Table 4 Comparison of examinees with and without sedation.

\begin{tabular}{|l|l|l|l|}
\hline & Sedation (yes) & Sedation (no) & P value \\
\hline Number of examinees & 208 & 105 \\
\hline Age (years) & $62.8 \pm 13.3$ & $63.7 \pm 13.8$ & 0.2933 \\
\hline Sex (M/F) & $107 / 101$ & $74 / 31$ & 0.0019 \\
\hline Scope (EG16-K10/EG17-J10) & $65 / 143$ & $39 / 66$ & 0.3586 \\
\hline Period (early/late) & $101 / 107$ & $49 / 56$ & 0.8443 \\
\hline TT (sec) & $228.7 \pm 70.8$ & $233.5 \pm 77.0$ & 0.2926 \\
\hline ET (sec) & $68.4 \pm 23.2$ & $72.6 \pm 26.6$ & 0.0725 \\
\hline GDT (sec) & $50.0 \pm 27.7$ & $50.9 \pm 18.7$ & 0.3801 \\
\hline ST (sec) & $110.4 \pm 49.3$ & $109.9 \pm 54.5$ & 0.4735 \\
\hline Number of pictures & $36.1 \pm 6.2$ & $36.6 \pm 7.0$ & 0.3336 \\
\hline Biopsy (Y/N) & $27 / 181(13.0 \%)$ & $9 / 96(8.6 \%)$ \\
\hline TT, total examination time; ET, esophageal insertion time; GDT, gastroduodenal insertion time; ST, surveillance time in the stomach.
\end{tabular}

- Table 5 Comparison of examinees older and younger than age 60 years.

\begin{tabular}{|l|l|l|l|}
\hline & Over $\mathbf{6 0}$ years & Under 60 years & P-value \\
\hline Number of examinees & 184 & 129 \\
\hline Age (years) & $72.5 \pm 7.0$ & $49.6 \pm 7.0$ & $<0.0001$ \\
\hline Gender (M/F) & $111 / 731$ & $70 / 59$ & 0.3407 \\
\hline Scope (EG16-K10/EG17-J10) & $59 / 125$ & $45 / 84$ & 0.6898 \\
\hline Period (early/late) & $88 / 96$ & $62 / 67$ & 1.0000 \\
\hline TT (sec) & $235.4 \pm 76.1$ & $223.1 \pm 67.7$ & 0.0720 \\
\hline ET (sec) & $70.7 \pm 27.5$ & $68.5 \pm 19.2$ & 0.2252 \\
\hline GDT (sec) & $51.1 \pm 28.4$ & $49.2 \pm 19.3$ & 0.2595 \\
\hline ST (sec) & $113.6 \pm 50.9$ & $105.3 \pm 50.9$ & 0.0795 \\
\hline Number of pictures & $36.6 \pm 6.9$ & $35.7 \pm 5.8$ & 0.1072 \\
\hline Biopsy (Y/N) & $28 / 156(15.2 \%)$ & $8 / 121(6.2 \%)$ \\
\hline TT, total examination time; ET, esophageal insertion time; GDT, gastroduodenal insertion time; ST, surveillance time in the stomach. \\
\hline
\end{tabular}

- Table 6 Analysis of the impact of patient age and type of scope used on examination times.

\begin{tabular}{|c|c|c|c|c|c|}
\hline & & & EG16-K10 & EG17-J10 & $P$ value \\
\hline \multirow[t]{6}{*}{ Age } & \multirow[t]{3}{*}{ Over 60 years } & $\mathrm{ET}(\mathrm{sec})$ & $78.6 \pm 27.9$ & $66.9 \pm 26.5$ & 0.0034 \\
\hline & & GDT (sec) & $56.2 \pm 42.7$ & $48.6 \pm 17.4$ & 0.0453 \\
\hline & & ST (sec) & $113.1 \pm 47.9$ & $113.9 \pm 52.4$ & 0.4581 \\
\hline & \multirow[t]{3}{*}{ Under 60 years } & $\mathrm{ET}$ (sec) & $72.5 \pm 17.4$ & $66.4 \pm 19.8$ & 0.0451 \\
\hline & & GDT (sec) & $53.9 \pm 21.6$ & $46.7 \pm 17.4$ & 0.0225 \\
\hline & & ST (sec) & $116.4 \pm 52.8$ & $99.4 \pm 48.8$ & 0.0357 \\
\hline
\end{tabular}

ET, esophageal insertion time; GDT, gastroduodenal insertion time; ST, surveillance time in the stomach. 
tis, which is removed by flushing water through the scope. For such examinees, higher insufflation-suction power, higher resolution of CCD, and a thicker insertion tube might be preferable to survey the lumen of the stomach in a shorter time.

In this study, we could not evaluate patient discomfort associated with scope insertion because some examinees underwent EGD using conscious sedation due to the style of our clinical daily practice. In addition, all examinees underwent EGD with transoral insertion. Therefore, we could not evaluate EGD with transnasal insertion. Evaluation of discomfort using a visual analog scale inr unsedated examinees undergoing both transoral and transnasal insertion is preferable, although the shorter examination time is obviously preferable to reduce discomfort associated with EGD. The examination time may be a surrogate marker for discomfort associated with EGD under the controlled condition of oral insertion.

Undoubtedly, the final objective of surveillance EGD is to detect all lesions that require treatment. Ultrathin endoscopes were not equipped with high-resolution CCD a decade ago, but they have now been significantly improved. In addition, newly developed image-enhancement technology has become available for ultrathin scopes [1-4]. In this study, we did not evaluate the lesion detection rate, although we found that the number of biopsies in examinees over 60 years old was significantly higher than in examinees under 60 years old ( $\downarrow$ Table 5 ). Considering the prevalence rate for lesions in elderly people, ultrathin endoscopes may be suitable for detecting lesions that require treatment.

The limitation of this study is its retrospective, single-institution design. Also, as mentioned above, we could not evaluate patient discomfort associated with transnasal EGD. Future studies are necessary to evaluate patient discomfort using a visual analog scale and compare examination time in a larger number of examinees.

\section{Conclusion}

In summary, this study demonstrated that use of an ultrathin endoscope with tapered body stiffness can reduce examination time by shortening insertion time. Although further analysis is needed of the correlation between examination time and patient discomfort associated, results of EGD with ultrathin endoscopes with tapering body stiffness were acceptable to both patients and operators.

\section{Acknowledgements}

The authors sincerely thank all co-medical staffs in the endoscopy unit of Chiba-Nishi General Hospital and Mr. Chinari Tanaka for their support and fruitful comments. The authors would like to thank Enago (www.enago.jp) for the English language review.

\section{Competing interests}

The authors declare that they have no conflict of interest.

\section{References}

[1] Muto M, Minashi K, Yano T et al. Early detection of superficial squamous cell carcinoma in the head and neck region and esophagus by narrow band imaging: a multicenter randomized controlled trial. J Clin Oncol 2010; 28: 1566-1572

[2] Osawa H, Yamamoto H. Present and future status of flexible spectral imaging color enhancement and blue laser imaging technology. Dig Endosc 2014; 26: (Suppl. 01): 105-115

[3] Neumann H, Fujishiro M, Wilcox CM et al. Present and future perspectives of virtual chromoendoscopy with i-scan and optical enhancement technology. Dig Endosc 2014; 1: 43-51

[4] Kodashima S, Fujishiro M, Ono $S$ et al. Evaluation of a new image-enhanced endoscopic technology using band-limited light for detection of esophageal squamous cell carcinoma. Dig Endosc 2014; 26: 164171

[5] Muto M, Yao K, Kaise M et al. Magnifying endoscopy simple diagnostic algorithm for early gastric cancer (MESDA-G). Dig Endosc 2016; 28: 379-393

[6] Yao K. Clinical application of magnifying endoscopy with narrowband imaging in the stomach. Clin Endosc 2015; 48: 481-490

[7] Uedo N, Takeuchi Y, Ishihara R. Endoscopic management of early gastric cancer: endoscopic mucosal resection or endoscopic submucosal dissection: data from a Japanese high-volume center and literature review. Ann Gastroenterol 2012; 25: 281-290

[8] Nagami Y, Ominami M, Otani K et al. Endoscopic submucosal dissection for adenocarcinomas of the esophagogastric junction. Digestion 2018; 97: 38-44

[9] Kakushima N, Yoshida M, Yabuuchi Y et al. Present status of endoscopic submucosal dissection for non-ampullary duodenal epithelial tumors. Clin Endosc 2020: doi:10.5946/ce.2019.184

[10] Tanuma T, Morita Y, Doyama H. Current status of transnasal endoscopy worldwide using ultrathin videoscope for upper gastrointestinal tract. Dig Endosc 2016; 28: 25-31

[11] Ono S, Niimi K, Fujishiro M et al. Evaluation of preferable insertion routes for esophagogastroduodenoscopy using ultrathin endoscopes. World J Gastroenterol 2014; 20: 5045-5050

[12] Ono S, Niimi K, Fujishiro M et al. Ultrathin endoscope flexibility can predict discomfort associated with unsedated transnasal esophagogastroduodenoscopy. World J Gastrointest Endosc 2013; 16: 346-351

[13] Sessler CN, Gosnell MS, Grap MJ et al. The Richmond Agitation-Sedation Scale: validity and reliability in adult intensive care unit patients. Am J Respir Crit Care Med 2002; 166: 1338-1344

[14] Sami SS, Subramanian V, Ortiz-Fernandez-Sordo J et al. Performance characteristics of unsedated ultrathin video endoscopy in the assessment of the upper GI tract: systematic review and meta-analysis. Gastrointest Endosc 2015; 82: 782-792 